\title{
Multiple domestications of Asian rice
}

To the Editor - In their recent Correspondence ${ }^{1}$ about our study showing that there were three origins of Asian rice $^{2}$, Huang and Han suggest that the methodology that we used to infer multiple domestications was flawed as it did not take account of the strong genetic bottleneck in japonica. We agree that the differences in diversity between japonica and the other groups present a challenge when comparisons are made, and we explored this issue in some detail in the Supplementary Information of our paper ${ }^{2}$.

Specifically, Huang and Han criticized our choice of a $\pi_{\text {(wild) }} / \pi_{\text {(domesticated) }}$ threshold of 4 for each of japonica, indica and aus, and implied that a higher threshold would be more appropriate for the detection of selective sweeps in japonica. We did, in fact, test the data with such an approach -it is described in the first paragraph of the Methods section of the paper, and the resulting tree is presented as Supplementary Fig. $1^{2}$. In brief, we compared the sweeps Huang et al. ${ }^{3}$ identified for indica at threshold 3 against the sweeps they identified for japonica at threshold 14 (Supplementary Section 2 of ref. 3) and extracted genomic regions that are detected as sweeps in both groups ('sweep overlaps'). The resulting tree reveals that even when a much higher sweep threshold is used for japonica (to reduce the swept fraction of the japonica genome), indica, japonica and aus still form separate clusters. We are therefore confident that the strong japonica bottleneck does not influence our results in the way suggested by Huang and Han ${ }^{1}$.

Huang and $\operatorname{Han}^{1}$ also contend that the presence of similar haplotypes, including identical coding sequences, in cultivated groups for loci such as sh 4 and PROG1 argues against multiple domestication. In the Supplementary Information of our paper ${ }^{2}$, we explain how selection of similar haplotypes that are geographically widespread within the standing variation can lead to this outcome, and we provide evidence indicating that the $\operatorname{sh} 4$ allele present in domesticated rice was selected from at least two wild sources. We do not believe that the 55 domestication sweeps previously identified by Huang et al. ${ }^{3}$ are validated by the presence within them of 'well-characterized domestication genes', as this assumes that these domestication genes have undergone selection in all groups of $O$. sativa, which has not been established for the majority of those genes. In fact, comparison of the selective sweeps separately detected for japonica and indica by Huang et al. (see Supplementary Tables 10 and 11 of ref. 3 ) shows that at least some of these genes have not undergone selection in both of these cultivated groups. For example the gSH1, Waxy and $R c$ genes, as well as various genomic regions associated with quantitative traits such as grain length, number and weight, tiller angle and panicle length, which are under strong selection in japonica, are not found within the selective sweeps of indica. This observation questions the use by Huang et al. ${ }^{3}$ of the single $O$. sativa supergroup for detection of the 55 'major domestication sweeps', as many regions passing the threshold can be under strong selection in one group but much weaker selection in another. Huang and Han admit that this happens: "the indica group has many allelic combinations at the 55 domestication loci but japonica has very few" ${ }^{\prime}$. It is indeed possible that the diversity of indica was enriched by japonica, but the multiplicity of the allele combinations indicates that these loci have been subject to zero or weak selection in indica. This suggests that such gene flow was not critical for the domestication of indica and probably occurred after the independent domestication of this group of rice.

\section{References}

1. Huang, X. \& Han, B. Nature Plants 2, 15207 (2015).

2. Civán, P., Craig, H., Cox, C. J. \& Brown, T. A. Nature Plants 1, 15164 (2015).

3. Huang, X. et al. Nature 490, 497-503 (2012).

Peter Civán', Hayley Craig', Cymon J. Cox² and Terence A. Brown ${ }^{1 *}$

${ }^{1}$ Manchester Institute of Biotechnology, Faculty of Life Sciences, University of Manchester, Manchester M1 7DN, UK. ${ }^{2}$ Centro de Ciências do Mar, Universidade do Algarve, Gambelas, 8005-139 Faro, Portugal.

*e-mail: terry.brown@manchester.ac.uk 